\title{
Generation and Spectroscopic Identification of the Thiuram Radical $\left(\mathrm{CH}_{3}\right)_{2} \mathrm{NCS}_{2}$.
}

Artur Mardyukov, * Felix Keul, and Peter R. Schreiner Institute of Organic Chemistry, Justus-Liebig University, Heinrich-Buff-Ring 17, 35392

Giessen, Germany; artur.mardyukov@org.chemie.uni-giessen.de

\section{Table of Contents}

Synthesis of deuterated tetramethylthiuram disulphide (Thiram-d6) ..................................2

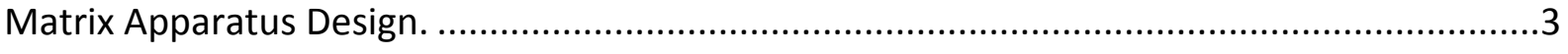

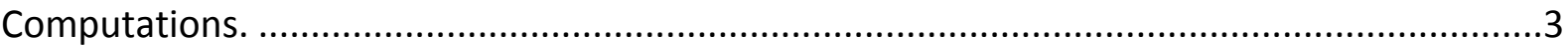

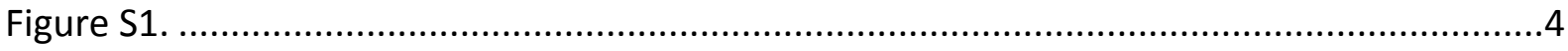

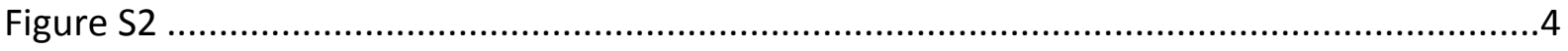

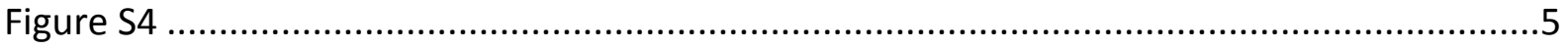

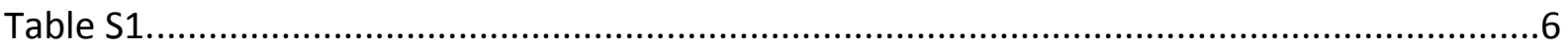

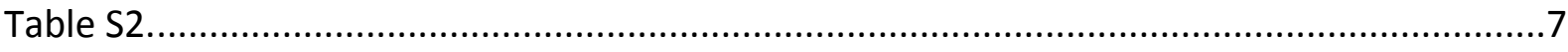

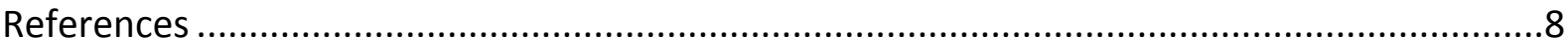

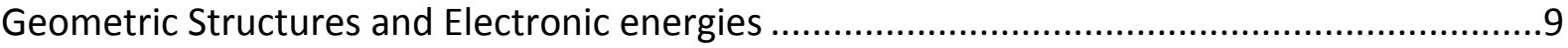




\section{Synthesis of deuterated tetramethylthiuram disulphide (Thiram-d6)}

$$
\begin{aligned}
& \text { 1) } 3.2 \text { equiv. } \mathrm{KOH}
\end{aligned}
$$

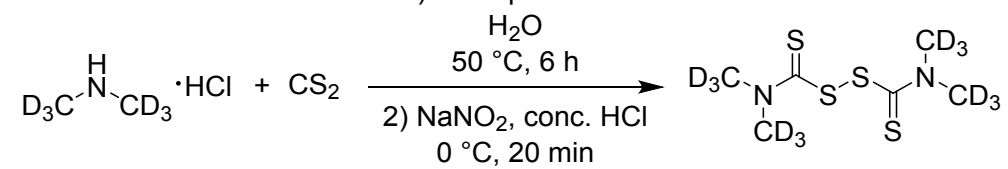

A mixture of deuterated dimethylamine- $d_{6}$-hydrochloride ( $400 \mathrm{mg}, 4.57 \mathrm{mmol}, 1.00$ equiv.), carbondisulfid ( $281 \mu \mathrm{L}, 4,66 \mathrm{mmol}, 1.02$ equiv.) and potassium hydroxide ( $821 \mathrm{mg}, 14.6 \mathrm{mmol}$, 3,20 equiv.) was dissolved in water and was heated at $50{ }^{\circ} \mathrm{C}$ under stirring for 6 hours. After this the reaction solution was cooled to $0{ }^{\circ} \mathrm{C}$ and $\mathrm{NaNO}_{2}(274 \mathrm{mg}, 3.97 \mathrm{mmol}, 0.87$ equiv.) in $274 \mu \mathrm{L} \mathrm{MeOH}$ was added. $1 \mathrm{~mL}$ concentrated $\mathrm{HCl}$ was added dropwise and the solution was stirred for another $20 \mathrm{~min}$. The reaction mixture was extracted three times with chloroform. The combined organic phases was tried over sodium sulfate and filtered. The filtrate was concentrated under reduced pressure and recrystallized from ethanol.

${ }^{13} \mathrm{C}-\mathrm{NMR}\left(100 \mathrm{MHz}, \mathrm{CDCl}_{3}\right): \delta / \mathrm{ppm}=194.0$

$\mathrm{IR}=\mathrm{u}\left(\mathrm{cm}^{-1}\right)=2058 ; 1674 ; 1432 ; 1260 ; 1207 ; 1109 ; 1055 ; 1015 ; 908 ; 887 ; 815 ; 768 ; 571 ; 530$

HR-MS-ESI: Calculated $[\mathrm{M}+\mathrm{H}]^{+}=275.0529 \mathrm{~m} / \mathrm{z}$, Found: $[\mathrm{M}+\mathrm{H}]^{+}=275.0526 \mathrm{~m} / \mathrm{z}$

The procedure was according to literature. ${ }^{1}$

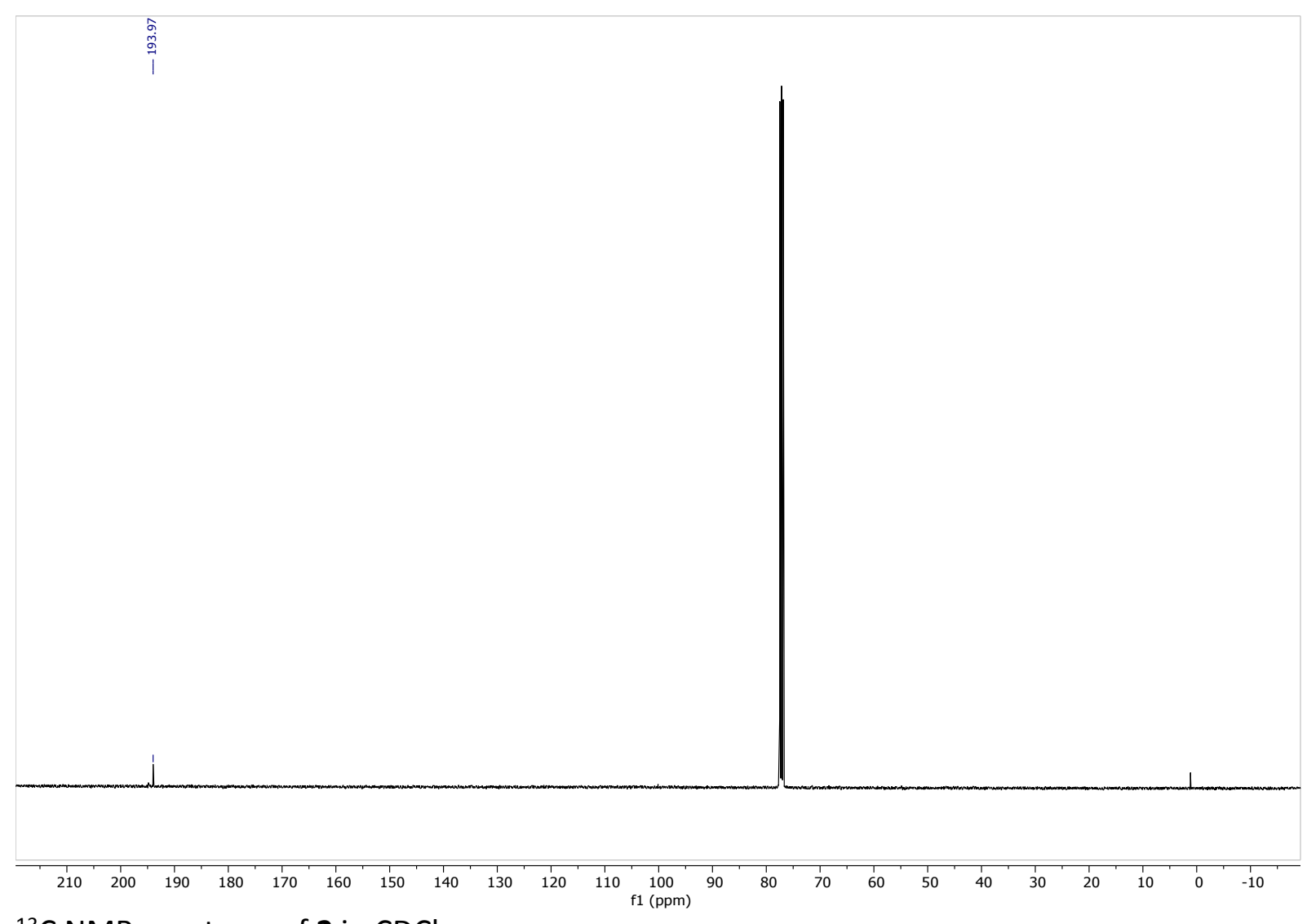

${ }^{13} \mathrm{C}$ NMR spectrum of 2 in $\mathrm{CDCl}_{3}$. 
Matrix Apparatus Design. For the matrix isolation studies, we used an APD Cryogenics HC-2 cryostat with a closed-cycle refrigerator system, equipped with an inner CsI window for IR measurements. Spectra were recorded with a Bruker IFS 55 FT-IR spectrometer with a spectral range of $4500-400 \mathrm{~cm}^{-1}$ and a resolution of $0.7 \mathrm{~cm}^{-1}$ and UV/Vis spectra were recorded with a JASCO V-670 spectrophotometer equipped with an inner sapphire window. Matrices were generated by co-deposition of 2 (evaporated at $150^{\circ} \mathrm{C}$ from a storage bulb) with a large excess of argon (typically 60-120 mbar from a $2000 \mathrm{~mL}$ storage bulb) on the surface of the matrix window at $10 \mathrm{~K}(20 \mathrm{~K})$. A high-pressure mercury lamp (HBO 200, Osram) with a monochromator (Bausch \& Lomb) was used for irradiation.

Computations. All geometries were optimized and characterized as minima or transition structures by means of analytical harmonic vibrational frequency computations at the B3LYP/6-311++G(3df,3pd) level of theory. ${ }^{2-4}$ All computations were performed with the Gaussian16 program. ${ }^{5}$

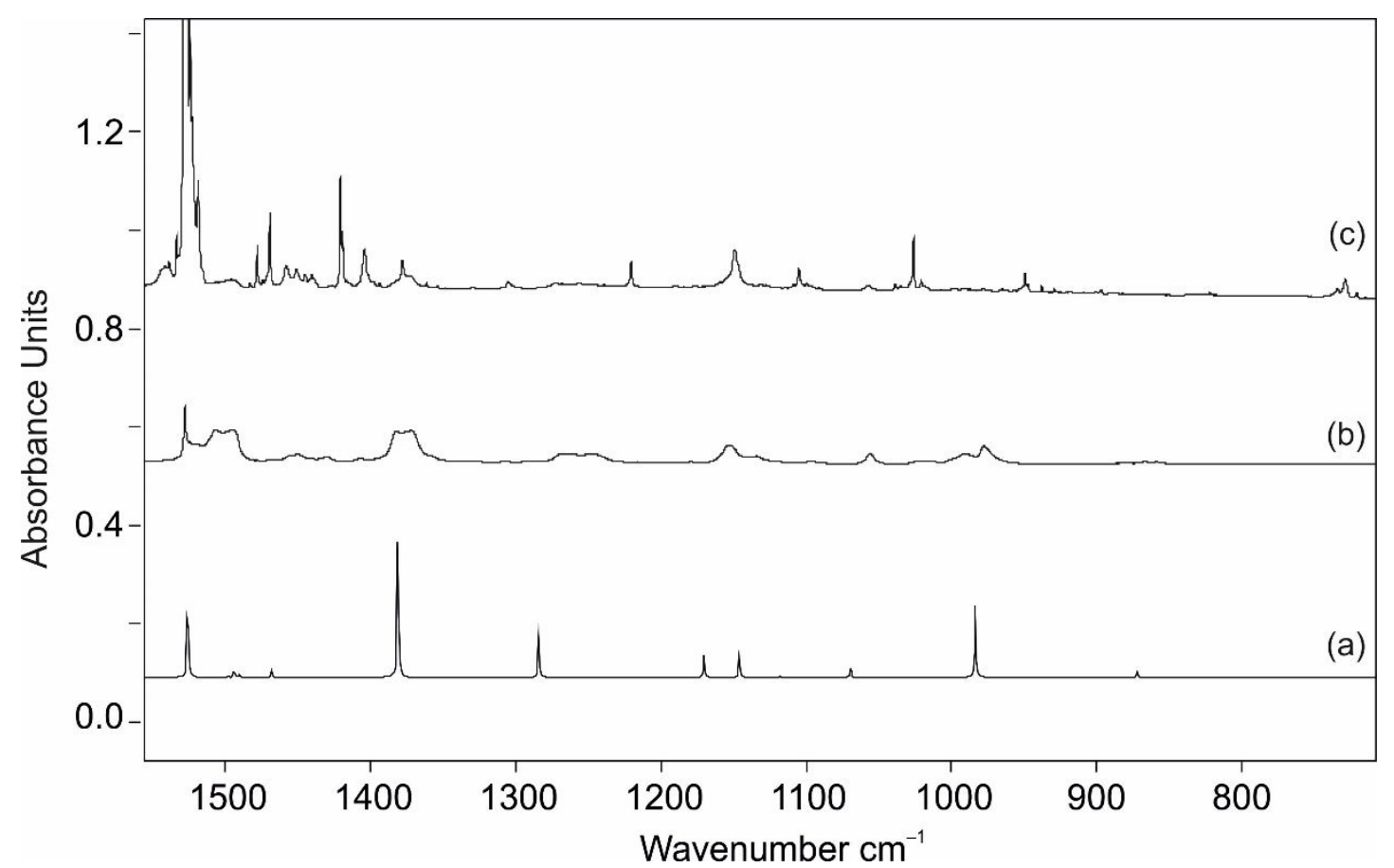

Figure S1. (a) IR spectrum of 2 computed at UB3LYP/6-311++G(3df,3pd) (unscaled). (b) IR spectrum of $\mathbf{2}$ in argon. (c) IR spectra showing the products of the pyrolysis of $\mathbf{2}$ in argon with subsequent trapping in an argon matrix at $10 \mathrm{~K}$. 


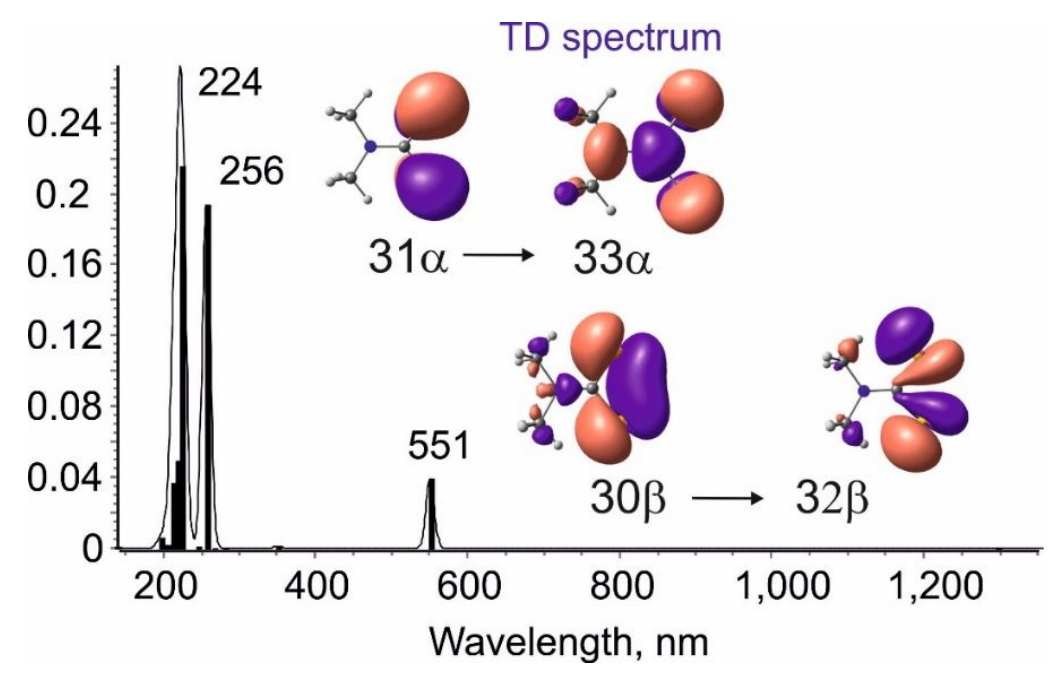

Figure S2. Computed [TD-UB3LYP/6-311++G(3df,3pd)] electronic transitions for 1. Molecular orbitals of $\mathbf{1}$ in its singlet ground state as computed at the UB3LYP/6$311++G(3 d f, 3 p d)$ level of theory.

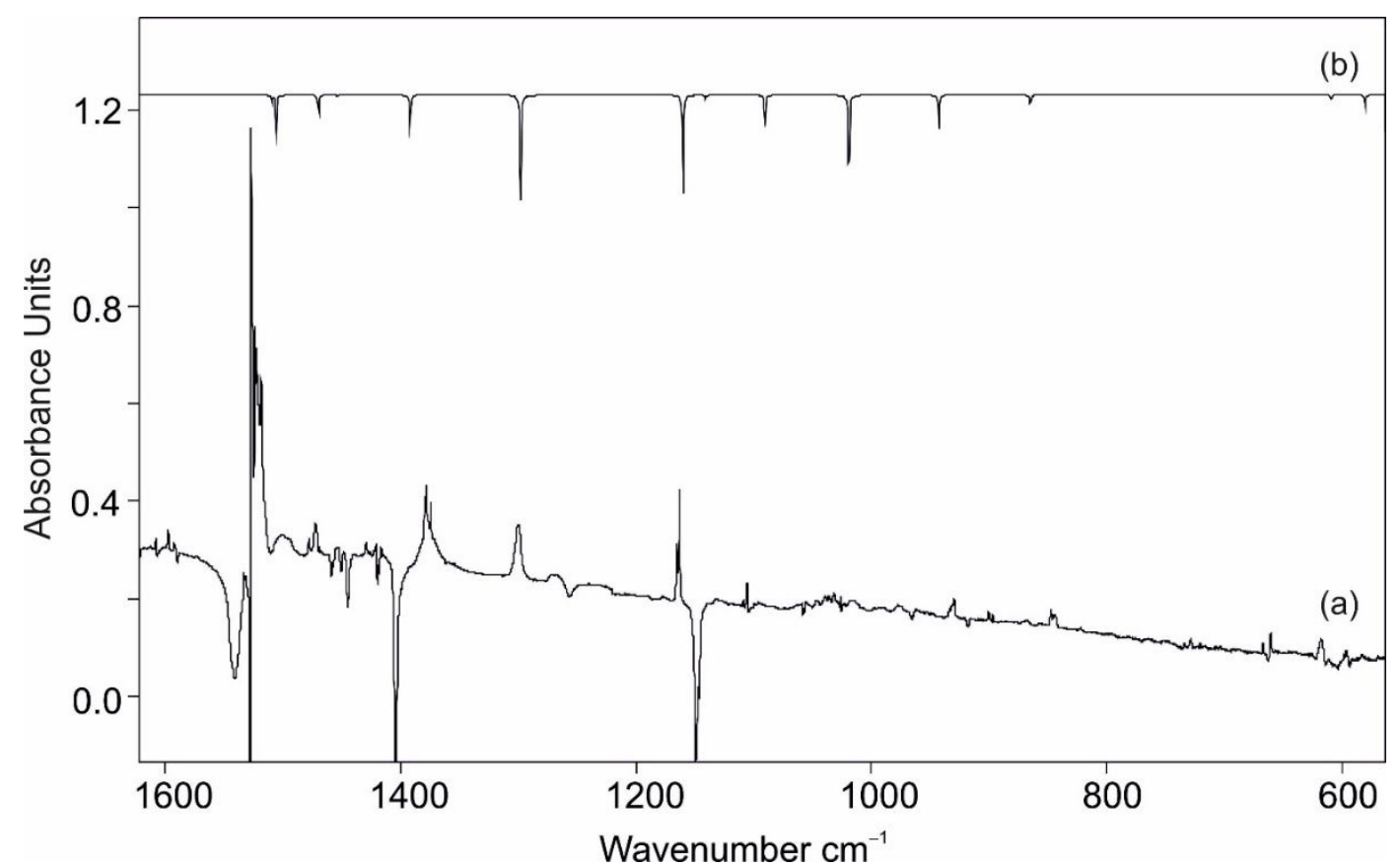

Figure S3. IR spectra showing the product of pyrolysis of $\mathbf{2}$ in argon matrix with subsequent trapping in an argon matrix at $10 \mathrm{~K}$. (a) IR difference spectra showing the photochemistry of 1 after irradiation with $\lambda=623 \mathrm{~nm}$ in argon at $10 \mathrm{~K}$. Downward bands assigned to 1 disappear while upward bands assigned to $\mathbf{3}$ appear after 5 min irradiation time. (b) IR spectrum of $\mathbf{3}$ computed at UB3LYP/6-311++G(3df,3pd) (unscaled). 


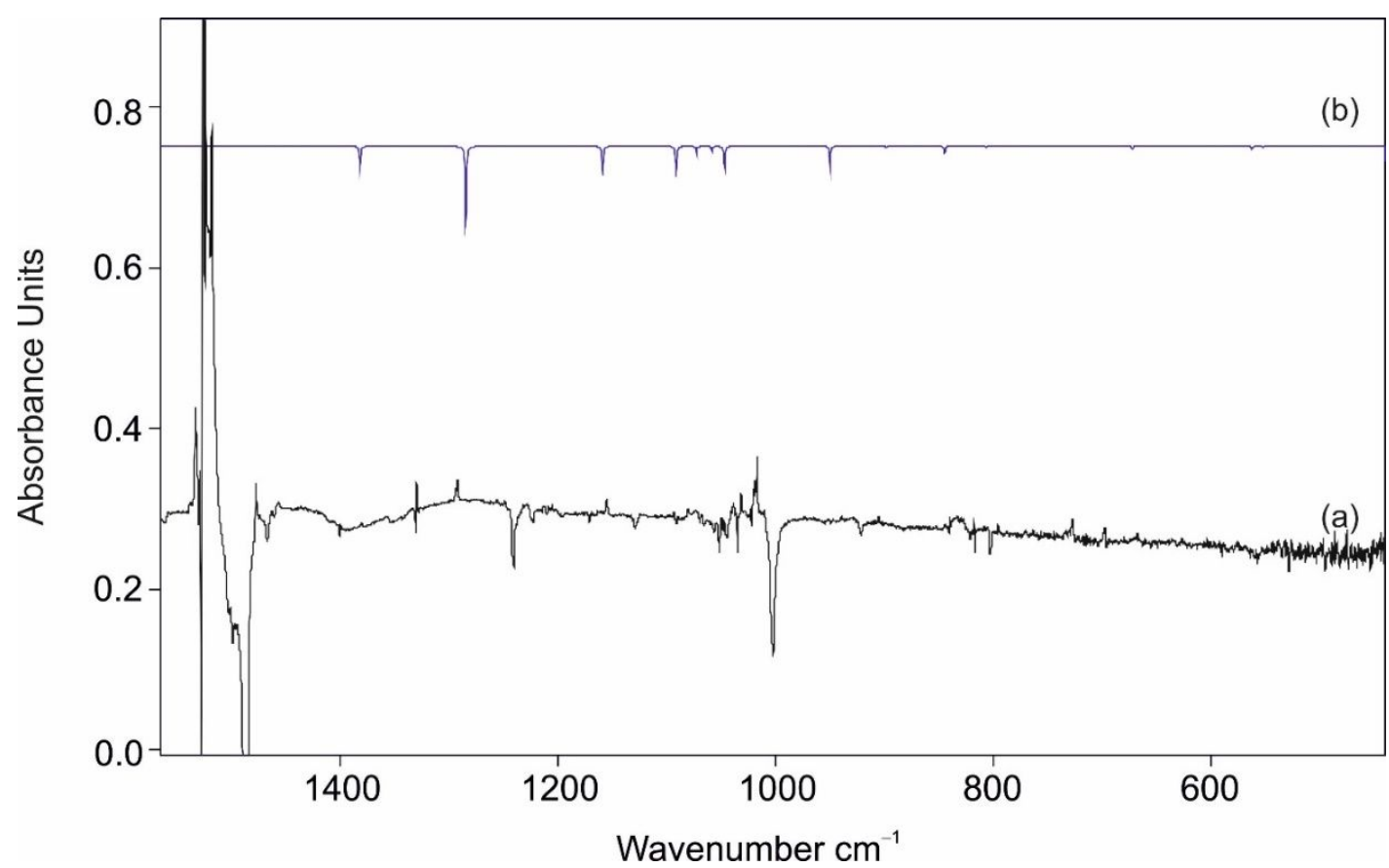

Figure S4. IR spectra showing the product of pyrolysis of $\mathbf{2}$ in argon matrix with subsequent trapping in an argon matrix at $10 \mathrm{~K}$. (a) IR difference spectra showing the photochemistry of $d_{6}-1$ after irradiation with $\lambda=623 \mathrm{~nm}$ in argon at $10 \mathrm{~K}$. Bands pointing downwards assigned to $d_{6}-\mathbf{1}$ disappear and bands pointing upwards assigned $d_{6}-\mathbf{3}$ appear after 5 min irradiation time. (b) IR spectrum of $d_{6}-3$ computed at UB3LYP/6-311++G(3df,3pd) (unscaled). 
Table S1. Experimental (Ar matrix, $10 \mathrm{~K}$ ) and computed IR frequencies of $\mathbf{1}$ and $d_{6}-\mathbf{1}$, band origins in $\mathrm{cm}^{-1}$, computed intensities $\left(\mathrm{km} \mathrm{mol}^{-1}\right)$ in parentheses.

\begin{tabular}{|c|c|c|c|c|c|}
\hline Mode & $\begin{array}{c}1 \\
\text { Computed }^{a} \\
\end{array}$ & $\begin{array}{c}1 \\
\mathrm{Ar}, 10 \mathrm{~K}^{\mathrm{b}} \\
\end{array}$ & $\begin{array}{c}d_{3}-\mathbf{1} \\
\text { Computed }^{\text {a }}\end{array}$ & $\begin{array}{r}d_{3}-1 \\
\text { Ar, } 10 K^{b} \\
\end{array}$ & Assignment \\
\hline 30 & $3140(0.0)$ & & $2326(0.1)$ & & $\mathrm{CH}$ str. \\
\hline 29 & 3136 (1.9) & & $2325(2.4)$ & & $\mathrm{CH}$ str. \\
\hline 28 & $3078(0)$ & & $2282(0)$ & & $\mathrm{CH}_{3}$ asym. str. \\
\hline 27 & 3076 (36.8) & 2971 & $2251(26.1)$ & 2069 & $\mathrm{CH}_{3}$ sym. str. \\
\hline 26 & 3025 (71.8) & 2922 & $2172(38.6)$ & 2042 & $\mathrm{CH}_{3}$ sym. str. \\
\hline 25 & 3021 (15) & & $2168(9.6)$ & & $\mathrm{CH}_{3}$ sym. str. \\
\hline 24 & $1563(180)$ & 1541 (s) & $1490(390.2)$ & 1485 (s) & CN str. \\
\hline 23 & 1493 (19.8) & $1457(\mathrm{~m})$ & $1153(0.9)$ & & $\mathrm{CH}_{3}$ def. \\
\hline 22 & $1492(13.3)$ & $1445(\mathrm{~m})$ & $1091(3.5)$ & & $\mathrm{CH}_{3}$ def. \\
\hline 21 & $1484(23.7)$ & & $1083(0.0)$ & & $\mathrm{CH}_{3}$ def. \\
\hline 20 & $1478(0)$ & & $1070(6.5)$ & & $\mathrm{CH}_{3}$ def. \\
\hline 19 & 1442 (18.9) & $1409(w)$ & 1072 (17.9) & $1052(w)$ & $\mathrm{CH}_{3}$ def. \\
\hline 18 & $1437(117.8)$ & $1404(s)$ & $1012(68.1)$ & $1003(\mathrm{~m})$ & $\mathrm{CH}_{3}$ def./C-N str. \\
\hline 17 & 1260 (21.9) & $1256(w)$ & $1239(22.6)$ & $1241(\mathrm{~m})$ & $\mathrm{CH}$ def. \\
\hline 16 & $1172(1.9)$ & & $924(1.6)$ & $902(w)$ & $\mathrm{CH}_{3}$ rocking \\
\hline 15 & $1166(148)$ & 1148 (s) & $830(1.8)$ & $821(w)$ & $\mathrm{CH}_{3}$ def./ $\mathrm{CN}$ str. \\
\hline 14 & $1117(0)$ & & $821(0)$ & & $\mathrm{CH}_{3}$ rocking \\
\hline 13 & $1070(3.2)$ & $1056(w)$ & $1043(0)$ & & $\mathrm{CH}_{3}$ rocking \\
\hline 12 & $971(8.4)$ & $965(w)$ & $965(4.3)$ & $921(w)$ & SCS asym str. \\
\hline 11 & $926(3.2)$ & $918(w)$ & $811(7.2)$ & $803(w)$ & C-N str. \\
\hline 10 & 594 (3.9) & $593(w)$ & $560(2.7)$ & $559(w)$ & breathing \\
\hline 9 & $545(0)$ & & 540 & & $\mathrm{C}-\mathrm{N}$ breathing \\
\hline 8 & 452 (10.5) & $453(\mathrm{~m})$ & $424(10.1)$ & $424(w)$ & distortion \\
\hline
\end{tabular}

aUB3LYP/6-311++G(3df,3pd), harmonic approximation, unscaled frequencies, intensities (in parentheses) in $\mathrm{km} \mathrm{mol}^{-1}$. ${ }^{\mathrm{b}}$ Experiment: argon matrix, $10 \mathrm{~K}$.; approximate relative intensities (w: weak, m: medium, s: strong). 
Table S2. Experimental (Ar matrix, $10 \mathrm{~K}$ ) and computed IR frequencies of $\mathbf{3}$ and $d_{3}-\mathbf{3}$, band origins in $\mathrm{cm}^{-1}$, computed intensities $\left(\mathrm{km} \mathrm{mol}^{-1}\right)$ in parentheses.

\begin{tabular}{|c|c|c|c|c|c|}
\hline Mode & $\begin{array}{l}3 \\
\text { Computed }^{a}\end{array}$ & $\begin{array}{l}3 \\
\mathrm{Ar}, 10 \mathrm{~K}^{\mathrm{b}}\end{array}$ & $\begin{array}{c}d_{6}-\mathbf{3} \\
\text { Computed }^{\mathrm{a}}\end{array}$ & $\begin{array}{r}d_{6}-3 \\
\text { Ar, } 10 \mathrm{~K}^{\mathrm{b}}\end{array}$ & Assignment \\
\hline 30 & $3330(0.4)$ & & $2489(0.3)$ & & $\mathrm{CH}_{2}$ asym. str. \\
\hline 29 & $3206(1.1)$ & & $2321(4.1)$ & & $\mathrm{CH}_{2}$ sym. str. \\
\hline 28 & $3159(3.6)$ & & $2345(2.1)$ & & $\mathrm{CH}_{3}$ asym. str. \\
\hline 27 & $3137(7)$ & & $2326(3.4)$ & & $\mathrm{CH}_{3}$ asym. str. \\
\hline 26 & 3064 (19.2) & & 2197 (13.4) & & $\mathrm{CH}_{3}$ sym. str. \\
\hline 25 & 2616 (11.6) & & $1887(5.5)$ & & S-H str. \\
\hline 24 & $1510(4.7)$ & & $1382(31.2)$ & $1330(\mathrm{~m})$ & $\mathrm{CH}_{2}$ def. \\
\hline 23 & $1506(27.3)$ & $1472(\mathrm{~m})$ & $1159(44.3)$ & & $\mathrm{CH}_{3}$ def. \\
\hline 22 & $1470(14.4)$ & $1454(w)$ & $1059(6.5)$ & $1155(w)$ & $\mathrm{CH}_{3}$ def./ $\mathrm{CH}_{2}$ def. \\
\hline 21 & $1454(0.9)$ & & 1047 (33.6) & & $\mathrm{CH}_{3}$ def.. \\
\hline 20 & $1392(27.4)$ & $1378(\mathrm{~s})$ & 1073 (11.9) & & $\mathrm{C}-\mathrm{N}$ str $/ \mathrm{CH}_{3}$ def. \\
\hline 19 & $1298(95.5)$ & 1300 (s) & $1285(128.1)$ & $1293(\mathrm{~m})$ & $\mathrm{NCH}$ def. \\
\hline 18 & $1160(65.8)$ & $1164(\mathrm{~s})$ & $1091(42.7)$ & $1083(w)$ & $\mathrm{CH}_{3}$ def./C-N str. \\
\hline 17 & $1141(2.3)$ & & $898(2)$ & & $\mathrm{CH}_{3}$ rocking \\
\hline 16 & $1091(21.8)$ & & $950(28.4)$ & $906(w)$ & $\mathrm{CH}_{3}$ rocking \\
\hline 15 & $1019(75)$ & & $807(1.6)$ & & CNC def \\
\hline 14 & $943(23.1)$ & $930(\mathrm{~m})$ & $673(5.2)$ & & CSH def. \\
\hline 13 & $865(8.2)$ & 847 (w) & 845 (9.8) & & CSH def. \\
\hline 12 & $609(3)$ & & $562(4)$ & & breathing. \\
\hline 11 & $581(8.1)$ & & $552(2)$ & & $\mathrm{C}-\mathrm{N}$ breathing \\
\hline 10 & $531(29)$ & 498 (m) & $417(21)$ & & $\mathrm{CH} 2$ o.o.p. def. \\
\hline 9 & $470(16.3)$ & $459(w)$ & $424(3.8)$ & & $\mathrm{CH}_{2}$ breathing \\
\hline 8 & $433(20.1)$ & & $332(10)$ & & distortion \\
\hline 7 & $420(3.2)$ & & $384(7)$ & & SH /CH o.o.p. def \\
\hline
\end{tabular}

a UB3LYP/6-311++G(3df,3pd), harmonic approximation, unscaled frequencies, intensities (in parentheses) in $\mathrm{km} \mathrm{mol}^{-1}$. ${ }^{\mathrm{b}}$ Experiment: argon matrix, $10 \mathrm{~K}$.; approximate relative intensities (w: weak, m: moderate, s: strong). 


\section{References}

(1) Kapanda, C. N.; Muccioli, G. G.; Labar, G.; Poupaert, J. H.; Lambert, D. M. Bis(dialkylaminethiocarbonyl)disulfides as Potent and Selective Monoglyceride Lipase Inhibitors. J. Med. Chem. 2009, 52, 7310-7314.

(2) Becke, A. D. Density-functional exchange-energy approximation with correct asymptotic behavior. Phys. Rev. A: Gen. Phys. 1988, 38, 3098-3100.

(3) Lee, C.; Yang, W.; Parr, R. G. Development of the Colle-Salvetti correlationenergy formula into a functional of the electron density. Phys. Rev. B: Condens. Matter 1988, 37, 785-789.

(4) Miehlich, B.; Savin, A.; Stoll, H.; Preuss, H. Results obtained with the correlation energy density functionals of Becke and Lee, Yang and Parr. Chem. Phys. Lett. 1989, 157, 200-206.

(5) Frisch, M. J.; Trucks, G. W.; Schlegel, H. B.; Scuseria, G. E.; Robb, M. A.; Cheeseman, J. R.; Scalmani, G.; Barone, V.; Petersson, G. A.; Nakatsuji, H.; Li, X.; Caricato, M.; Marenich, A.; Bloino, J.; Janesko, B. G.; Gomperts, R.; Mennucci, B.; Hratchian, H. P.; Ortiz, J. V.; Izmaylov, A. F.; Sonnenberg, J. L.; Williams-Young, D.; Ding, F.; Lipparini, F.; Egidi, F.; Goings, J.; Peng, B.; Petrone, A.; Henderson, T.; Ranasinghe, D.; Zakrzewski, V. G.; Gao, J.; Rega, N.; Zheng, G.; Liang, W.; Hada, M.; Ehara, M.; Toyota, K.; Fukuda, R.; Hasegawa, J.; Ishida, M.; Nakajima, T.; Honda, Y.; Kitao, O.; Nakai, H.; Vreven, T.; Throssell, K.; Montgomery, J., J. A.; Peralta, J. E.; Ogliaro, F.; Bearpark, M.; Heyd, J. J.; Brothers, E.; Kudin, K. N.; Staroverov, V. N.; Keith, T.; Kobayashi, R.; Normand, J.; Raghavachari, K.; Rendell, A.; Burant, J. C.; Iyengar, S. S.; Tomasi, J.; Cossi, M.; Millam, J. M.; Klene, M.; Adamo, C.; Cammi, R.; Ochterski, J. W.; Martin, R. L.; Morokuma, K.; Farkas, O.; Foresman, J. B.; Fox, D. J. Gaussian, Inc., Wallingford CT, 2016. 


\section{Geometric Structures and Electronic energies}

2: Thiram

\begin{tabular}{|c|c|c|c|}
\hline \multicolumn{4}{|c|}{01} \\
\hline 6 & -2.179240000 & -0.262350000 & -0.019838000 \\
\hline & -3.315196000 & 0.467150000 & -0.052663000 \\
\hline & -3.309224000 & 1.926078000 & 0.009643000 \\
\hline & -2.550378000 & 2.335850000 & -0.656663000 \\
\hline & -4.275387000 & 2.297757000 & -0.318264000 \\
\hline & -3.117402000 & 2.288270000 & 1.022821000 \\
\hline & -4.603697000 & -0.218927000 & 0.016307000 \\
\hline & -4.722217000 & -0.734149000 & 0.970670000 \\
\hline & -5.393177000 & 0.518099000 & -0.098888000 \\
\hline & -4.677517000 & -0.964297000 & 2832000 \\
\hline 16 & -2.145830000 & -1.924642000 & 0.004081000 \\
\hline 16 & -0.712553000 & 54000 & 72000 \\
\hline 16 & 0.712553000 & -0.766754000 & -0.009786000 \\
\hline 6 & 2.179240000 & 0.262350000 & \\
\hline & 3.315196000 & -0.467 & 0.052669000 \\
\hline 6 & & & -0.016286000 \\
\hline & 5.393176000 & -0.518099000 & 0.098916000 \\
\hline & 4.722227000 & 0.734151000 & -0.970648000 \\
\hline & 4.677509000 & 0.964296000 & 0.772855000 \\
\hline & 3.309223000 & -1.926078000 & -0.009638000 \\
\hline & 3.117402000 & -2.288268000 & -1.022818000 \\
\hline & 4.275386000 & -2.297758000 & 0.318269000 \\
\hline 1 & 2.550376000 & -2.335851000 & 0.656666000 \\
\hline 16 & 2.145830000 & 1.924642000 & -0.004083000 \\
\hline \multicolumn{4}{|c|}{$E[B 3 L Y P]=-1938.3661847$} \\
\hline \multicolumn{4}{|c|}{ ZPVE[B3LYP] = 0.182309} \\
\hline
\end{tabular}

1: Thiuram radical $-\left(C_{2 v}\right.$ point group $)$

$\begin{array}{lrrr}02 & & & \\ 6 & 0.000000000 & 0.000000000 & -0.258622000 \\ 7 & 0.000000000 & 0.000000000 & 1.074375000 \\ 6 & 0.000000000 & 1.231043000 & 1.850148000 \\ 1 & 0.000000000 & 2.086260000 & 1.181504000 \\ 1 & -0.887212000 & 1.271609000 & 2.484642000 \\ 1 & 0.887212000 & 1.271609000 & 2.484642000 \\ 6 & 0.000000000 & -1.231043000 & 1.850148000 \\ 1 & 0.887212000 & -1.271609000 & 2.484642000 \\ 1 & -0.887212000 & -1.271609000 & 2.484642000 \\ 1 & 0.000000000 & -2.086260000 & 1.181504000 \\ 16 & 0.00000000 & 1.365482000 & -1.264758000 \\ 16 & 0.000000000 & -1.365482000 & -1.264758000\end{array}$

$E[B 3 L Y P]=-969.1687156$

ZPVE[B3LYP] $=0.090156$ 
3: ( $C_{1}$ point group)

$\begin{array}{lccc}02 & & & \\ 6 & 0.250547000 & 0.143603000 & 0.009758000 \\ 7 & -1.006159000 & -0.411084000 & 0.002044000 \\ 6 & -2.152995000 & 0.498290000 & 0.170728000 \\ 1 & -1.956161000 & 1.190217000 & 0.983162000 \\ 1 & -2.308796000 & 1.072536000 & -0.739900000 \\ 1 & -3.031286000 & -0.101171000 & 0.388579000 \\ 6 & -1.285058000 & -1.726142000 & -0.200159000 \\ 16 & 0.537529000 & 1.774636000 & -0.085800000 \\ 16 & 1.661941000 & -0.920751000 & 0.057227000 \\ 1 & 1.102996000 & -2.012485000 & 0.617333000 \\ 1 & -2.309957000 & -2.040357000 & -0.129618000 \\ 1 & -0.520170000 & -2.387808000 & -0.558648000\end{array}$

$E[B 3 L Y P]=-969.1311047$

ZPVE $[B 3 L Y P]=0.086126$

4: thiyl radical

02

$\begin{array}{llll}6 & 0.089205000 & 0.465870000 & -0.000002000\end{array}$

$\begin{array}{lllll}16 & 1.569024000 & -0.075668000 & 0.000000000\end{array}$

$\begin{array}{llll}16 & -1.468691000 & -0.162005000 & 0.000000000\end{array}$

$\begin{array}{llll}1 & -2.140560000 & 1.007545000 & 0.000006000\end{array}$ $E[B 3 L Y P]=-835.1037877$

ZPVE $[B 3 L Y P]=0.014078$

5: methanimine

01

$\begin{array}{lrrr}7 & 0.140674000 & -0.529853000 & -0.000001000 \\ 6 & -1.147352000 & 0.133151000 & 0.000000000 \\ 1 & -1.711569000 & -0.189293000 & -0.876245000 \\ 1 & -1.711561000 & -0.189281000 & 0.876254000 \\ 1 & -1.077911000 & 1.228629000 & -0.000007000 \\ 6 & 1.180571000 & 0.180278000 & 0.000000000 \\ 1 & 1.160825000 & 1.277636000 & -0.000002000 \\ 1 & 2.156177000 & -0.299289000 & 0.000002000 \\ \text { E[B3LYP] = -133.9946837 } & \\ \text { ZPVE[B3LYP] }=0.067886 & \\ 6 & & \\ 6: \text { dimethyl amine radical } & \\ 02 & & & \\ 7 & 0.000000000 & 0.000000000 & 0.629500000 \\ 6 & 0.000000000 & 1.192610000 & -0.168865000 \\ 1 & 0.000000000 & 2.077407000 & 0.465163000 \\ 1 & 0.879916000 & 1.230404000 & -0.827612000\end{array}$ 


$\begin{array}{rrrr}1 & -0.879916000 & 1.230404000 & -0.827612000 \\ 6 & 0.000000000 & -1.192610000 & -0.168865000 \\ 1 & 0.879916000 & -1.230404000 & -0.827612000 \\ 1 & 0.000000000 & -2.077407000 & 0.465163000 \\ 1 & -0.879916000 & -1.230404000 & -0.827612000\end{array}$

$E[B 3 L Y P]=-134.562047$

ZPVE $[B 3 L Y P]=0.076769$

CS2

01

$\begin{array}{llll}6 & 0.000000000 & 0.000000000 & 0.000000000\end{array}$

$\begin{array}{llll}16 & 0.000000000 & 0.000000000 & 1.553545000\end{array}$

$\begin{array}{llll}16 & 0.000000000 & 0.000000000 & -1.553545000\end{array}$

$E[B 3 L Y P]=-834.5686089$

$\mathrm{ZPVE}[\mathrm{B} 3 \mathrm{LYP}]=0.006957$

TS1:

02

$\begin{array}{llll}6 & -0.337722000 & -0.059178000 & -0.037192000 \\ 7 & 1.030835000 & -0.147230000 & -0.074555000 \\ 6 & 1.724197000 & -1.410811000 & 0.141383000 \\ 1 & 2.251558000 & -1.390115000 & 1.097600000 \\ 1 & 0.983379000 & -2.206373000 & 0.155428000 \\ 1 & 2.440091000 & -1.587864000 & -0.659574000 \\ 6 & 1.777969000 & 1.036719000 & -0.137233000 \\ 16 & -1.411148000 & -1.318149000 & -0.057875000 \\ 16 & -0.896768000 & 1.587235000 & 0.087181000 \\ 1 & 0.541100000 & 1.916492000 & 0.292801000 \\ 1 & 2.683408000 & 1.044477000 & 0.458298000 \\ 1 & 1.824605000 & 1.548228000 & -1.093301000\end{array}$

$E[B 3 L Y P]=-969.1137301$

ZPVE[B3LYP] $=0.083331$

TS-2:

02

$\begin{array}{lccc}6 & 0.596607000 & 0.178958000 & 0.311399000 \\ 7 & -1.317500000 & -0.259819000 & -0.068223000 \\ 6 & -2.199038000 & 0.842714000 & 0.245753000 \\ 1 & -2.010269000 & 1.651460000 & -0.458683000 \\ 1 & -3.248514000 & 0.545827000 & 0.204385000 \\ 1 & -1.955117000 & 1.218450000 & 1.237991000 \\ 6 & -1.748558000 & -1.421089000 & -0.307220000 \\ 16 & 0.965499000 & 1.685385000 & -0.160601000 \\ 16 & 1.496927000 & -1.267649000 & 0.022998000 \\ 1 & 0.991116000 & -2.014500000 & 1.026976000 \\ 1 & -2.810318000 & -1.667346000 & -0.275255000 \\ 1 & -1.037277000 & -2.202429000 & -0.555796000 \\ \text { E[B3LYP] =-969.0828436 } & \\ \text { ZPVE[B3LYP] }=0.083634 & \end{array}$


TS3:

02

$\begin{array}{llcc}6 & -0.802303000 & 0.121168000 & 0.000124000 \\ 7 & 1.090850000 & -0.459911000 & 0.000657000 \\ 6 & 1.842948000 & -0.369496000 & -1.221239000 \\ 1 & 1.168026000 & -0.353550000 & -2.074248000 \\ 1 & 2.487063000 & 0.515724000 & -1.249732000 \\ 1 & 2.493527000 & -1.248164000 & -1.306309000 \\ 6 & 1.844523000 & -0.364150000 & 1.221188000 \\ 1 & 2.494469000 & -1.242952000 & 1.309665000 \\ 1 & 2.489392000 & 0.520663000 & 1.244657000 \\ 1 & 1.170673000 & -0.343550000 & 2.074940000 \\ 16 & -1.759911000 & -1.143354000 & 0.001251000 \\ 16 & -0.568221000 & 1.708734000 & -0.001501000 \\ \text { E[B3LYP] =-969.1042532 } & \\ \text { ZPVE[B3LYP] }=0.085335 & \end{array}$

